requires a signalling input that is influenced by necroptosis.

Necroptosis is a more inflammatory form of cell death than apoptosis. The authors analysed immune-signalling molecules called cytokines in the livers of mice given the cancer-inducing treatments, and observed differences in the cytokine pattern depending on whether HCC or ICC developed. For example, they noted an increase in the expression of cytokines AIMP1, CCL4 and CXCL13 associated with ICC, compared with their levels in HCC. This raised the question of whether necroptosis-associated inflammation might induce epigenetic changes in hepatocytes that are poised to become cancer cells. The authors found differences in chromatin structure in selected regions of the genome between the two cancer subtypes, although when and how these differences arose is unknown.

Seehawer and colleagues also observed that the transcription-factor protein TBX3 was more highly expressed in HCC than in ICC, whereas the transcription factor PRDM5 had the opposite pattern of higher expression in ICC than in HCC. These expression differences were associated with epigenetic differences in the chromatin structure of the genes encoding these proteins. Remarkably, when the authors analysed samples of human HCC or ICC, they also observed the same pattern of higher TBX3 expression in HCC than in ICC, and higher PRDM5 expression in ICC than in HCC.

Altogether, the evidence suggests that the cell-death conditions prevailing in the liver at the earliest stages of tumour formation might account for the formation of these two different tumour subtypes. Early events in tumour formation are long over by the time a biopsy sample is taken from a human liver; this mouse study could help to illuminate key events that shape the initial steps of tumour formation and result in different cancer identities.

The study provides strong evidence that the tumour microenvironment provides yet-to-be identified signals that can impart long-lasting changes to the fate of cells poised to form cancer cells. Perhaps cytokines might be the drivers of this cancer-subtype switch. If so, the release of such cytokines because of tissue damage or disease might shape the identity of a cancer that is starting to form nearby.

In Seehawer and colleagues' experimental system, the cancer-subtype switch occurred after the acquisition of cancer-promoting genes. However, in human cancers, a different sequence of events is often noted that is thought to occur before the acquisition of cancer-promoting genetic changes. In this scenario, an increased risk of cancer is associated with a process called metaplasia, in which one type of differentiated cell reversibly switches to a different type of differentiated cell. How a predisposition to malignancy arises because of metaplasia is unknown.

It has been reported ${ }^{8}$ that the abnormal accumulation of bile-duct-like structures in the livers of mice that have chronic liver disease is not due to the proliferation of bileduct cells, as was previously thought, but that these structures arise from hepatocytes, and that this therefore constitutes a form of metaplasia. Interestingly, a similar pattern of growth of bile-duct-like cells is often observed in human livers ${ }^{8,9}$. Although this is not usually considered as a form of metaplasia, it is associated with an increase in the risk of liver cancer ${ }^{9}$. Could the switch between HCC and ICC in mice be similar to the process that occurs in liver metaplasia in humans? If it is, Seehawer and colleagues' work might provide insight into how metaplasia increases cancer risk. -

Eli Pikarsky is at the Lautenberg Center

for Immunology and Cancer Research,
IMRIC, and in the Department of Pathology,

The Hebrew University of Jerusalem,

Jerusalem 9112102, Israel.

e-mail:peli@hadassah.org.il

1. Seehawer, M. et al. Nature 562, 69-75 (2018).

2. Van Keymeulen, A. et al. Nature 525, 119-123 (2015).

3. Koren, S. et al. Nature 525, 114-118 (2015).

4. Yang, J. et al. Cell 117, 927-939 (2004).

5. Llovet, J. M. et al. Nature Rev. Dis. Primers 2, 16018 (2016).

6. Fan, B. et al. J. Clin. Invest. 122, 2911-2915 (2012).

7. Sekiya, S. \& Suzuki, A. J. Clin. Invest. 122 3914-3918 (2012).

8. Tarlow, B. D. et al. Cell Stem Cell 15, 605-618 (2014).

9. Ziol, M. et al. Gastroenterology 139, 335-343 (2010).

This article was published online on 12 September 2018.

\title{
IMMUNOLOGY
}

\section{Put to sleep by immune cells}

The sleep disorder narcolepsy is linked to immune-system genes and is caused by the loss of neurons that express the protein hypocretin. Hypocretin-targeting immune cells have now been found in people with narcolepsy. SEE ARTICLE P.63

\section{ROLAND S. LIBLAU}

卫 The events that lead to the sleep disorder narcolepsy are a long-standing mystery. On page 63, Latorre et al. ${ }^{1}$ reveal that people with narcolepsy have unusually high levels of a type of immune cell called a T cell, which targets proteins normally present in neurons in the brain. This finding raises the question of whether narcolepsy arises because $\mathrm{T}$ cells unleash an autoimmune response against neurons that are important for sleep regulation.

Narcolepsy affects around 1 in 2,000 people ${ }^{2}$. The symptoms usually begin in adolescence or early adulthood, and include daytime sleepiness and, in some cases, cataplexy - sudden muscle weakness during wakefulness that causes falls. A small population of neurons in the brain produces a protein called hypocretin, which controls sleep-wake cycles ${ }^{3}$, and narcolepsy-like symptoms occur in animals that have defects in genes required for the production of or response to hypocretin ${ }^{4}$. Narcolepsy type 2 is associated with daytime sleepiness, and this can progress to narcolepsy type 1 , which is characterized by sleepiness and cataplexy. People with narcolepsy type 1 have abnormally low numbers of hypocretinproducing neurons ${ }^{5}$.

Hypocretin levels in the cerebrospinal fluid that bathes the brain and spinal cord can be measured to help diagnose $e^{6}$ narcolepsy type 1 ,

and such tests provide a way of indirectly monitoring the loss of hypocretin-producing neurons over time. The trajectory of this neuronal loss remains to be fully understood, but can take months or years.

Studies of human genetics have implicated immune-system genes in narcolepsy. Yet whether the immune system contributes to the demise of hypocretin-producing neurons, and if so, how, was unknown. HLA genes encode proteins that can present protein fragments called antigens to $\mathrm{T}$ cells, and this interac-

"Do T cells that target neuronal proteins other than hypocretin have a role in narcolepsy?"

tion can trigger an immune response against cells that contain the specific antigen. Autoimmune diseases are often associated with HLA genes $^{7}$. A version of one such gene, called $H L A-D Q B 1^{\star} 06: 02$, is present in more than $98 \%$ of people with narcolepsy ${ }^{8}$, but it is found in only $15-30 \%$ of the general population, depending on ethnicity ${ }^{8,9}$. Moreover, previous reports ${ }^{10,11}$ suggest that antibodies targeting neuronal proteins are present at a higher than usual frequency in people with narcolepsy.

Latorre and colleagues used various techniques to identify human $\mathrm{T}$ cells that recognize specific antigens. The authors tested whether $\mathrm{T}$ cells that recognize antigens from hypocretin were present in blood samples 
from 19 people with narcolepsy (14 of whom had $\left.H L A-D Q B 1^{*} 06: 02\right)$ and from a control group of 13 people who didn't have narcolepsy and carried the $H L A-D Q B 1^{*} 06: 02$ gene variant. The authors found that all of those with narcolepsy had a type of T cell called a CD4 ${ }^{+}$ memory $\mathrm{T}$ cell that gave a substantial response to peptide fragments of hypocretin, and this response included the expression of immunesignalling molecules called cytokines (Fig. 1). However, only three members of the control group had T cells that responded to hypocretin, and this response was much weaker than that of the narcolepsy group. When the authors analysed samples of $\mathrm{CD} 4^{+} \mathrm{T}$ cells, they found that the proportion of these cells that recognize hypocretin was more than ten times higher in individuals with narcolepsy than in control individuals. This increased T-cell reactivity to hypocretin has also been reported independently ${ }^{12}$, and strongly suggests that autoimmunity has a role in narcolepsy.

The authors' in-depth in vitro analysis of the samples from people with narcolepsy enabled the specific hypocretin peptides recognized by the T cells to be characterized, together with the versions of the $\mathrm{T}$-cell receptors that were involved in antigen recognition. Unexpectedly, most of these T cells did not recognize hypocretin peptides bound to the HLA-DQ6 proteins that are encoded by $H L A-D Q B 1^{*} 06: 02$. Instead, HLA proteins called HLA-DR were involved.

Several explanations for this are possible. Maybe an initial T-cell response to hypocretin-producing neurons is dominated by $\mathrm{T}$ cells that recognize antigens bound to HLA-DQ6, but over time the response shifts to $T$ cells that recognize antigens presented on HLA-DR. An observation consistent with this model is the recent report ${ }^{12}$ that the proportion of $\mathrm{T}$ cells that respond to hypocretin presented by HLA-DQ6 is higher than normal in people with recent-onset narcolepsy. Another possibility is that $\mathrm{T}$ cells in the bloodstream (tested by the authors) have different HLAbinding preferences from those in the brain. Or perhaps $H L A-D Q B 1^{*} 06: 02$ might help to promote the development of a repertoire of $T$ cells that recognize antigens in association with HLA-DR proteins ${ }^{13}$.

The authors made the puzzling finding that immune cells called antigen-presenting cells, which, as their name indicates, can present antigens to $\mathrm{T}$ cells, did not cleave hypocretin into the specific peptides to which the T cells responded. This suggests that hypocretin needs to be cleaved, either extracellularly or in neurons that express hypocretin, to enable presentation of these peptides by antigenpresenting cells.

Previous epidemiological studies ${ }^{14,15}$ have revealed an increased risk of narcolepsy in people who were vaccinated against the 2009 H1N1 strain of influenza virus. Crossreactivity can occur if a $\mathrm{T}$ cell recognizes antigens from

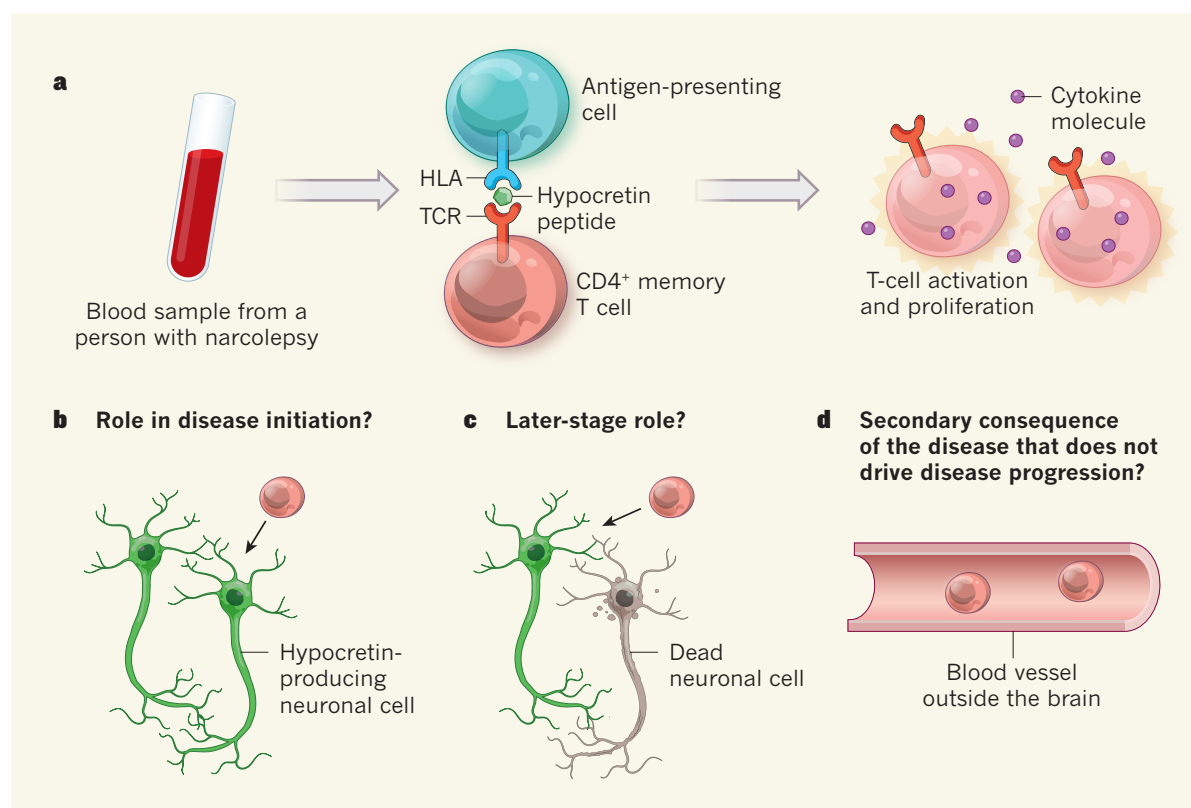

Figure 1 | Immune cells and narcolepsy. The sleep disorder narcolepsy is caused by loss of brain cells that produce the protein hypocretin. a, Latorre et al. ${ }^{1}$ analysed blood samples from 19 people with narcolepsy. They stimulated a type of immune cell in the samples, called a CD4 $4^{+}$memory T cell, with antigen-presenting cells, which had an HLA protein displaying a peptide fragment of hypocretin protein on their surfaces. In all the samples, this type of $\mathrm{T}$ cell recognized the antigen when the antigen was bound to the T-cell receptor (TCR). This recognition led to T-cell proliferation and activation, and the production of immune-stimulating molecules called cytokines. Whether and, if so, how such hypocretin-targeting T cells contribute to narcolepsy is unknown. $\mathbf{b}$, One possibility is that they have a role in the processes that cause disease onset and aid the initial killing of hypocretin-producing neurons. c, Or perhaps these T cells contribute to the processes that kill neurons as the disease progresses. d, Another possibility is that the presence of these $T$ cells is a secondary consequence of the disease (if, for instance, they are generated in an immune response induced by neuronal death), but these $\mathrm{T}$ cells do not contribute to disease progression because, for example, they do not have access to hypocretinproducing neurons.

different types of protein that have some structural similarity ${ }^{16}$. Latorre et al. report that the $\mathrm{T}$ cells they tested that can recognize hypocretin do not recognize antigens from the H1N1 flu virus, and thus do not show crossreactivity. The nature of the link between vaccination against the $2009 \mathrm{H} 1 \mathrm{~N} 1 \mathrm{flu}$ strain and the narcolepsy cases that arose postvaccination remains unknown.

There is a dearth of information about the immunological processes that occur in the brain during narcolepsy. Latorre and colleagues analysed samples of cerebrospinal fluid from seven people with narcolepsy. One of them had hypocretin-recognizing $\mathrm{T}$ cells of a type termed $\mathrm{CD} 8^{+}$. Further studies are therefore needed to determine whether T cells that recognize hypocretin are enriched in the cerebrospinal fluid of people who have narcolepsy.

Latorre and colleagues noted the presence of T cells that recognize hypocretin in blood samples of two people with narcolepsy type 2 who lacked $H L A-D Q B 1^{*} 06: 02$, and in whom hypocretin levels were not abnormally low. This challenges the model that targeting of hypocretin by such $\mathrm{T}$ cells is involved in neuronal destruction. However, the authors did detect a high frequency of hypocretin-specific $\mathrm{CD}^{+}$ $\mathrm{T}$ cells in blood samples from a person with narcolepsy type 2 who subsequently developed cataplexy. Therefore, it is possible that this person's hypocretin-producing neurons had been undergoing a process of destruction. This observation is consistent with results from a mouse study showing that $\mathrm{CD} 8^{+} \mathrm{T}$ cells that recognize an antigen expressed by hypocretin-producing neurons can directly kill these neurons ${ }^{17}$.

Several questions remain to be answered. For example, do T cells that target neuronal proteins other than hypocretin have a role in narcolepsy? What are the contributions of $\mathrm{CD} 4^{+}$and $\mathrm{CD} 8^{+} \mathrm{T}$ cells, and are other immune cells or antibodies involved? $\mathrm{CD}^{+} \mathrm{T}$ cells recognize antigens bound to HLA proteins that belong to a group called HLA class I, which can be present on neurons. However, if $\mathrm{CD}^{+} \mathrm{T}$ cells target neurons, it is unclear how this occurs, because neurons do not express HLA class II proteins (a group that includes HLA-DQ6 and HLA-DR) that CD $4^{+} \mathrm{T}$ cells bind to ${ }^{18}$.

Firmly establishing whether there is a causal relationship between the presence of hypocretin-targeting $\mathrm{T}$ cells and narcolepsy is a key issue, because if this is confirmed it would provide a therapeutic target for narcolepsy. Some insights might come from studying immune responses over time in 


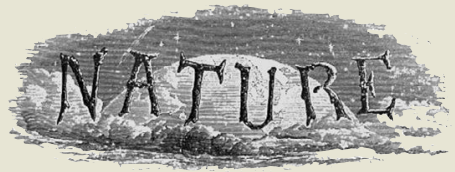

50 Years Ago

A chain of ecological events extraordinary for the British Isles is reported in this week's issue of Nature ... They concern the unusual bloom of a planktonic alga, the dinoflagellate Gonyaulax tamarensis, off the Northumbrian coast of Britain in May this year. This eventually led to the illness of more than eighty people through mussel poisoning and the deaths of about 80 per cent of the breeding population of shags on the Farne Islands ... Fortunately for the mussel eaters of Northumberland, most of the mussels eaten had been well cooked, the juices had been drained away, and in this way some of the poison was eliminated. Otherwise there would probably have been much more serious effects and even some deaths.

From Nature 5 October 1968

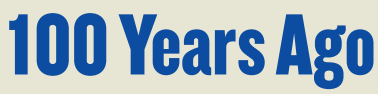

In North Wales, on August 20 ... I saw a rainbow-effect which was quite new to me. The summit of Tryfaen ... has three sharp, rocky peaks ... We had climbed up the eastern cliff in a south-westerly gale, which brought up much cloud with some light showers, and were sitting just below the top of the southern peak. The Holyhead road lay north-east and $2000 \mathrm{ft}$. below us. From it rose the upright portion of a brilliant rainbow. At the centre of its circle was the shadow of our peak with those of the other two peaks to the left of it, all sharply defined. Around the shadow of our peak was a most vivid and persistent bow, the smallest I have ever seen, the radius of the inner edge being about half that of the outer ... Outside this bow (which had the colours in regular rainbow order, red outside) was part of a third bow of perhaps double the diameter, but dim and intermittent.

From Nature 3 October 1918 patients, ideally starting at disease onset.

It would indeed be valuable to obtain a precise picture of the contribution of hypocretin-specific $\mathrm{CD} 4^{+} \mathrm{T}$ cells to narcolepsy. Do these cells initiate the disease-causing process? Do they contribute to the later-stage progression? Are they present as a secondary consequence of the disease but do not contribute to disease progression? There are many possibilities to consider. For example, do unidentified immune cells cause the demise of hypocretin-producing neurons and lead to a second wave of immune cells, such as hypocretin-specific T cells, that target the neurons? Or perhaps such second-wave T cells might not contribute to the progression of narcolepsy at all, because they might not interact with hypocretin-producing neurons.

If further experiments strengthen the proposed link between increased T-cell reactivity to hypocretin and neuronal damage, Latorre and colleagues' study might lead to targeted immune therapies. If this is the case, such treatments would probably be developed to target the immune system at the time of onset of narcolepsy.

Roland S. Liblau is at the Center for Physiopathology of Toulouse Purpan, University of Toulouse, CNRS, INSERM, Toulouse III University, Toulouse 31024, France. e-mail:roland.liblau@inserm.fr
1. Latorre, D. et al. Nature 562, 63-68 (2018).

2. Scammell, T. E. N. Engl. J. Med. 373, 2654-2662 (2015).

3. de Lecea, L. et al. Prog. Brain Res. 198, 15-24 (2012).

4. Sakurai, T. Curr. Opin. Neurobiol. 23, 760-766 (2013).

5. Liblau, R. S., Vassalli, A., Seify, A. \& Tafti, M. Lancet Neurol. 14, 318-328 (2015)

6. Dauvilliers, Y., Arnulf, I. \& Mignot, E. Lancet 369 499-511 (2007).

7. Dendrou, C. A., Petersen, J., Rossjohn, J. \& Fugger, L Nature Rev. Immunol. 18, 325-339 (2018).

8. Behalf of the European Narcolepsy Network (EU-NN). Sleep 37, 19-25 (2014).

9. Mignot, E. et al. Am. J. Hum. Genet. 68, 686-699 (2001).

10.Cvetkovic-Lopes, V. et al. J. Clin. Invest. 120, 713-719 (2010).

11.Ahmed, S. S. et al. Sci. Transl. Med. 7, 294 ra105 (2015).

12.Luo, G. et al. Preprint at bioRxiv https://doi. org/10.1101/378109 (2018).

13.Sharon, E. et al. Nature Genet. 48, 995-1002 (2016).

14.Nguyen, X. H., Saoudi, A. \& Liblau, R. S. Curr. Opin Neurol. 29, 362-371 (2016).

15. Sarkanen, T. O., Alakuijala, A. P. E., Dauvilliers, Y. A \& Partinen, M. M. Sleep Med. Rev. 38, 177-186 (2018).

16. Birnbaum, M. E. et al. Cell 157, 1073-1087 (2014).

17. Bernard-Valnet, R. et al. Proc. Natl Acad. Sci. USA 113, 10956-10961 (2016).

18. Liblau, R. S., Gonzalez-Dunia, D., Wiendl, H. \& Zipp, F. Trends Neurosci. 36, 315-324 (2013).

The author declares competing financial interests. See go.nature.com/2nsaOol for details.

This article was published online on 12 September 2018.

\section{Morphing into action}

An organic polymer exhibits a phase transition that is associated with improved electromechanical properties. This feature links organic polymers with widely used perovskite materials, and could have many applications. SEE LETTER P.96

\section{RONALD E. COHEN}

$\mathrm{T}$ he properties of a material can change dramatically in the vicinity of a phase transition. For example, the electromechanical properties of ferroelectric materials are greatly enhanced in a transition region known as a morphotropic phase boundary. Here, the material's electric polarization (dipole moment per unit volume) rotates from one direction to another. This phenomenon is well studied in perovskite materials, but on page 96 , Liu et al. ${ }^{1}$ report similar behaviour in an organic polymer. The discovery could open up various applications, such as in medical instruments, power-generating clothes and improved safety devices for structures and vehicles.

Active materials transform energy from one type into another. For example, ferroelectrics convert electrical energy into mechanical energy, and vice versa. Ferroelectrics are used in technologies ranging from medical ultrasound systems to fuel-injection and crashsensing equipment in cars. They even have applications in refrigeration and the generation of power from excess heat.

The most commonly used ferroelectric is the perovskite lead zirconate titanate (PZT). Although PZT is cheap, it contains toxic lead and is a hard ceramic that can be produced only at high temperatures. The lead content limits the material's use in applications such as tiny pumps, and motors that are permanently installed in the human body. Singlecrystal 'relaxor' ferroelectrics have also been developed. These materials have much higher energy-conversion efficiencies than PZT and are advancing the resolution of medical ultrasound systems, for example. But they are much more expensive than PZT, and also contain lead.

In addition, there are active materials based on lead-free polymers. These materials can 